\title{
BMJ Open Study protocol for a randomised, double-blind, placebo-controlled clinical trial of duloxetine for the treatment and prevention of musculoskeletal pain: altering the transition from acute to chronic pain (ATTAC pain)
}

\author{
Daniel H Strauss, ${ }^{1}$ Divya R Santhanam, ${ }^{2}$ Samuel A McLean, ${ }^{3}$ \\ Francesca L Beaudoin ${ }^{4,5}$
}

To cite: Strauss DH, Santhanam DR, McLean SA, et al. Study protocol for a randomised, double-blind, placebo-controlled clinical trial of duloxetine for the treatment and prevention of musculoskeletal pain: altering the transition from acute to chronic pain (ATTAC pain). BMJ Open 2019;9:e025002. doi:10.1136/ bmjopen-2018-025002

- Prepublication history for this paper is available online. To view these files, please visit the journal online (http://dx.doi. org/10.1136/bmjopen-2018025002)

Received 25 June 2018 Revised 3 December 2018 Accepted 22 January 2019

D) Check for updates

(C) Author(s) (or their employer(s)) 2019. Re-use permitted under CC BY-NC. No commercial re-use. See rights and permissions. Published by BMJ.

For numbered affiliations see end of article.

\section{Correspondence to} Dr Francesca L Beaudoin; Francesca_Beaudoin@brown. edu

\section{ABSTRACT}

Introduction Chronic musculoskeletal pain affects a substantial portion of adults visiting the emergency department (ED). Current treatment is limited in scope and does not effectively reduce musculoskeletal pain in patients. The study will evaluate the use of duloxetine, a serotonin-norepinephrine reuptake inhibitor Food and Drug Administration approved for the treatment of chronic pain, as a promising option in its prevention. The proposed study may present a well-tolerated and effective non-opioid treatment for patients with acute musculoskeletal pain that may also be effective in preventing the transition to persistent or chronic musculoskeletal pain.

Methods and analysis The primary outcome of this study will be to assess the tolerability and preliminary effectiveness of duloxetine in patients with acute musculoskeletal pain. The study will take place at two EDs in Rhode Island, USA. The study will involve randomisation to one of three arms: duloxetine $30 \mathrm{mg}$, duloxetine $60 \mathrm{mg}$ or placebo. Tolerability will be assessed by comparing the proportion of participants that report an adverse event and that drop-out across the three study arms. Effectiveness will be determined by self-reported pain over 6 weeks of follow-up. Specifically, we will compare the proportion of participants with persistent pain (ongoing pain at 6-week follow-up), across the three study arms. 60 adults (aged 18-59) presenting to the ED with acute axial musculoskeletal pain within 7 days of onset are expected to be enrolled in the proposed study.

Ethics and dissemination Ethics approval was obtained by the Institutional Review Board (IRB). These results will be published in a peer reviewed scientific journal and presented at one or more scientific conferences.

Trial registration number NCT03315533.

\section{INTRODUCTION}

Chronic musculoskeletal pain affects $30 \%$ of adults, ${ }^{1}$ and the costs associated with treating
Strengths and limitations of this study

Findings of this study will address an urgent gap in non-opioid management options.

- Double blind randomised controlled trial is the most rigorous study design to address this research question.

Potentially may have a high dropout rate.

chronic pain are immense, totalling over $\$ 220$ billion in direct health costs. ${ }^{2}$ Musculoskeletal pain is one of the most common reasons to visit the emergency department (ED) with persistent and minor chronic pain presenting in a substantial proportion of patients $(>20 \%)$. Individuals with chronic musculoskeletal pain often report injury as an inciting event, ${ }^{3}$ and epidemiological studies have indeed demonstrated that chronic musculoskeletal pain commonly develops after common events such as motor vehicle collisions (MVCs) and episodes of acute low back pain. ${ }^{4-7}$ Our preliminary work suggests that current mainstays of treatment, opioids or non-steroidal anti-inflammatory drugs (NSAIDs), do little to reduce musculoskeletal pain persistence. ${ }^{8}$ Current treatment of acute musculoskeletal pain with opioids has numerous limitations, including pain worsening (opioid-induced hyperalgaesia), increased risk of transition to chronic musculoskeletal pain, functional decline, dependence and/or abuse. ${ }^{910}$

There is an urgent need for non-opioid pain management options to reduce acute 
pain and prevent the development of chronic musculoskeletal pain. This proposed altering the transition from acute to chronic pain (ATTAC pain) study will help address this critical need by evaluating the preliminary tolerability and effectiveness of a non-opioid, duloxetine-a serotonin-norepinephrine reuptake inhibitor (SNRI) marketed for the treatment of generalised anxiety disorder, major depressive disorder and chronic pain. Evidence suggests that stress systems and their interactions with neurological and immune systems play an influential role in persistent and chronic musculoskeletal pain development; serotonin and norepinephrine are important neurotransmitters in this regard. ${ }^{11}{ }^{12}$ The ATTAC pain study will examine whether duloxetine can reduce acute and persistent musculoskeletal pain among adults presenting to the ED with either traumatic or atraumatic (eg, lifting injury) acute musculoskeletal pain.

Duloxetine is an ideal candidate intervention because (1) a large body of data support the efficacy of duloxetine in chronic pain conditions ${ }^{13}{ }^{14}$; (2) duloxetine targets neurological, stress and immune mechanisms that play a critical role in chronic musculoskeletal pain development ${ }^{15}$; (3) duloxetine has been shown to reduce opioid use in other acute musculoskeletal pain settings ${ }^{16}$; (4) duloxetine reduces post-traumatic stress disorder (PTSD) symptoms in trauma survivors, ${ }^{17}$ and PTSD has been shown to contribute to functional decline, ${ }^{18}$ opioid/ substance abuse ${ }^{19}$ and chronic musculoskeletal pain development ${ }^{20}$; (5) duloxetine is well-demonstrated to be safe $^{21}$; and (6) duloxetine is a generic, low cost medication $(\$ 15-30 /$ month).

The primary aims of the proposed study will (1) assess the tolerability of duloxetine in patients with acute musculoskeletal pain by measuring the proportion of participants who report an adverse event $(\mathrm{AE})$ in the duloxetine versus placebo groups, as well as the overall drop-out rate in each study arm. Secondary outcomes of interest are: (2) the potential effectiveness of duloxetine in improving pain outcomes in patients with acute musculoskeletal pain will be indicated by examining whether duloxetine: reduces acute musculoskeletal pain symptoms (during the first 2 weeks after the ED visit), decreases the amount of rescue analgesia needed by participants after their ED visit and decreases persistent musculoskeletal pain incidence and severity 6 weeks after the ED visit.

\section{METHODS}

\section{Trial design}

This study is a randomised, double-blind, placebo-controlled clinical trial with three study arms. Patients will either receive $30 \mathrm{mg}$ duloxetine, $60 \mathrm{mg}$ duloxetine or a placebo medication.

\section{Settings}

This study will be carried out in the EDs of Rhode Island Hospital (RIH) and The Miriam Hospital, both in Providence, Rhode Island. RIH is a large, tertiary care, academic, level-1 trauma centre with more than 105000 adult ED visits per year. The Miriam Hospital is a community-based academic hospital with more than 65000 ED visits per year. Recruitment is expected to begin in February 2018 and continue for a period of about 12-18 months. We used the Standard Protocol Items: Recommendations for Interventional Trials checklist when writing our report.

\section{Recruitment and consent}

Patients presenting to the ED will be screened by study research assistants (RAs) for potential eligibility. This study will use a convenience sample of patients who present to the ED when study RAs are available, although the times of non-coverage are rare. Using the electronic medical record (EMR) system, reports will be run on a biweekly basis to identify potential missed enrolments at each of the study sites. RAs will be stationed in the ED and review the EMR for relevant clinical history such as the chief symptom, injury history and medical history pertaining to the inclusion/exclusion criteria listed below. After the screening process is completed, if the patient is willing and eligible to participate, then written informed consent will be obtained. After informed consent has been obtained, a pregnancy test will be performed (if needed). The patient history, screening data and any other test results (eg, pregnancy test) will then be reviewed with an Medical doctor investigator. This investigator will then give final eligibility approval to proceed with randomisation and will be available by cell phone $24 / 7$ to discuss eligibility in real time.

\section{Study population}

Patients at Rhode Island and Miriam Hospital EDs presenting with axial musculoskeletal pain (in the back, neck or shoulder region) with pain onset in the last 7 days will be included in the study. ${ }^{22} \mathrm{~A}$ shorter duration of acute pain ( $<7$ days) was chosen for two reasons: first ED patients with acute pain, are likely to present early in the course of pain and second, a hypothesised mechanism by which duloxetine may prevent chronic pain (descending modulation) may be dependent on pain duration. ${ }^{23}$ If this preliminary study is effective, subsequent studies may examine different durations of pain intensity as a moderator of treatment effect. Patients that also satisfy the following inclusion and exclusion criteria displayed in table 1 will be eligible to participate in the study.

\section{Randomisation and study intervention}

The active drug arms will consist of duloxetine extended release capsules. Active drug dosing will consist of either $30 \mathrm{mg}$ or $60 \mathrm{mg}$ per dose daily for 14 days starting in the ED. Study medication, including both duloxetine and placebo, will be compounded by the Investigational Drug Service (IDS) pharmacy at each of the study sites. All medication will be compounded in batches and packaged in bulk containers for storage at the IDS pharmacy. The containers will be labelled with a lot number 
Table 1 Inclusion and exclusion criteria

\begin{tabular}{|c|c|}
\hline Inclusion criteria & Exclusion criteria \\
\hline $\begin{array}{l}\text { Age } 18 \text { to } 59 \\
\text { Alert and oriented } \\
\text { Axial musculoskeletal pain (back, neck, shoulders) } \\
\text { Present to the Emergency Department (ED) with acute } \\
\text { (present for } \leq 7 \text { days) musculoskeletal pain and have a } \\
\text { current pain score of } \geq 4\end{array}$ & 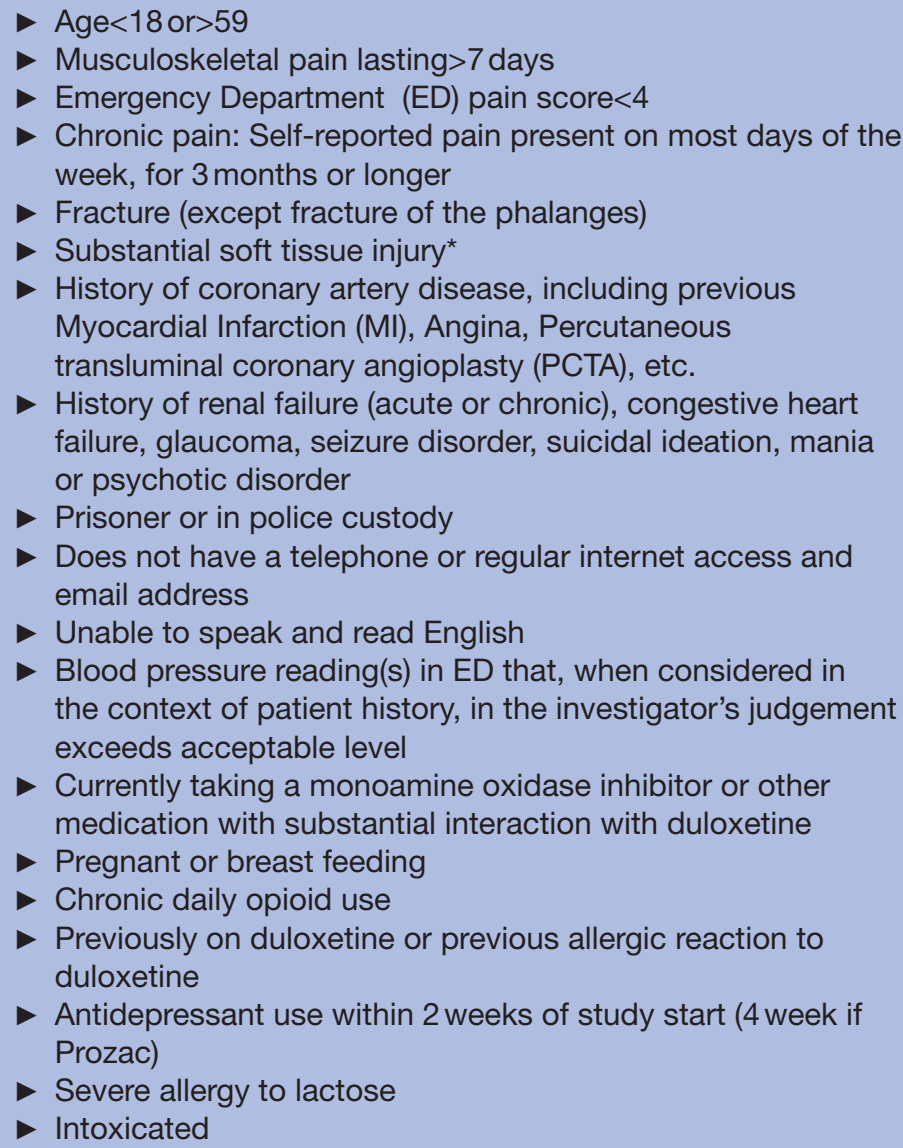 \\
\hline
\end{tabular}

*Giant abrasion: road rash to a surface area that is great than $15 \times 15 \mathrm{~cm}$ in area. Large avulsion-type injury: skin with or without subcutaneous tissue torn off an area of skin $>5 \times 5 \mathrm{~cm}$. Giant laceration: lacerations greater than $20 \mathrm{~cm}$ in length. Many lacerations: greater than four lacerations requiring sutures. Burn: any partial thickness burn $>3 \mathrm{~cm}^{2}$.

and expiration date and stored at room temperature. Once a patient's history and screening results have been cleared by the study investigator, the participant will be randomised by the study site IDS to receive duloxetine (30 $\mathrm{mg}$ or $60 \mathrm{mg}$ ) versus placebo, using a randomisation schedule with permuted block sizes stratified on gender and the type of musculoskeletal pain (traumatic or atraumatic) as the type of pain/injurymay be predictive of whether or not the participant responds to treatment. At the time of patient discharge from the study site, the study site IDS will dispense a 14-day supply of study medication. This medication will be prescribed by the treating physician. Participants will be offered a copy of the package insert for duloxetine.

\section{Assessments}

All study data are collected by direct data entry using REDCap software. This software is located on a secure server at RIH. This system is password protected and protected by backup to hard drive. If the internet at an ED site is temporarily unavailable, data will be collected via back-up paper forms and subsequently entered into the database. The study team has expertise in the use of such software for previous randomised controlled trials (RCT). Identifying information will only be kept until a patient has completed all follow-up evaluations, after which it will be destroyed. Paper survey forms will be labelled with participant study identification number only.

During the ED visit, participants will complete survey questions regarding somatic and psychological symptoms, ${ }^{24} 25$ pain and pain-related symptoms, ${ }^{26}$ general health and medication history, ${ }^{27}$ drug use ${ }^{28}$ and a demographic questionnaire. To increase adherence to the protocol, participants will be directed to download an optional MediSafeapplication onto their smartphone to serve as a daily reminder to take to study medication. Standard procedures to operationalise methods to reduce the magnitude of the placebo effect will also be taken. This is valuable to increase study power, as the placebo effect generally biases study results toward the null. ${ }^{29} 30$ These methods include: using patient reported ratings (investigator cannot cause inflation of scores); excluding 
Table 2 Study assessments and timing

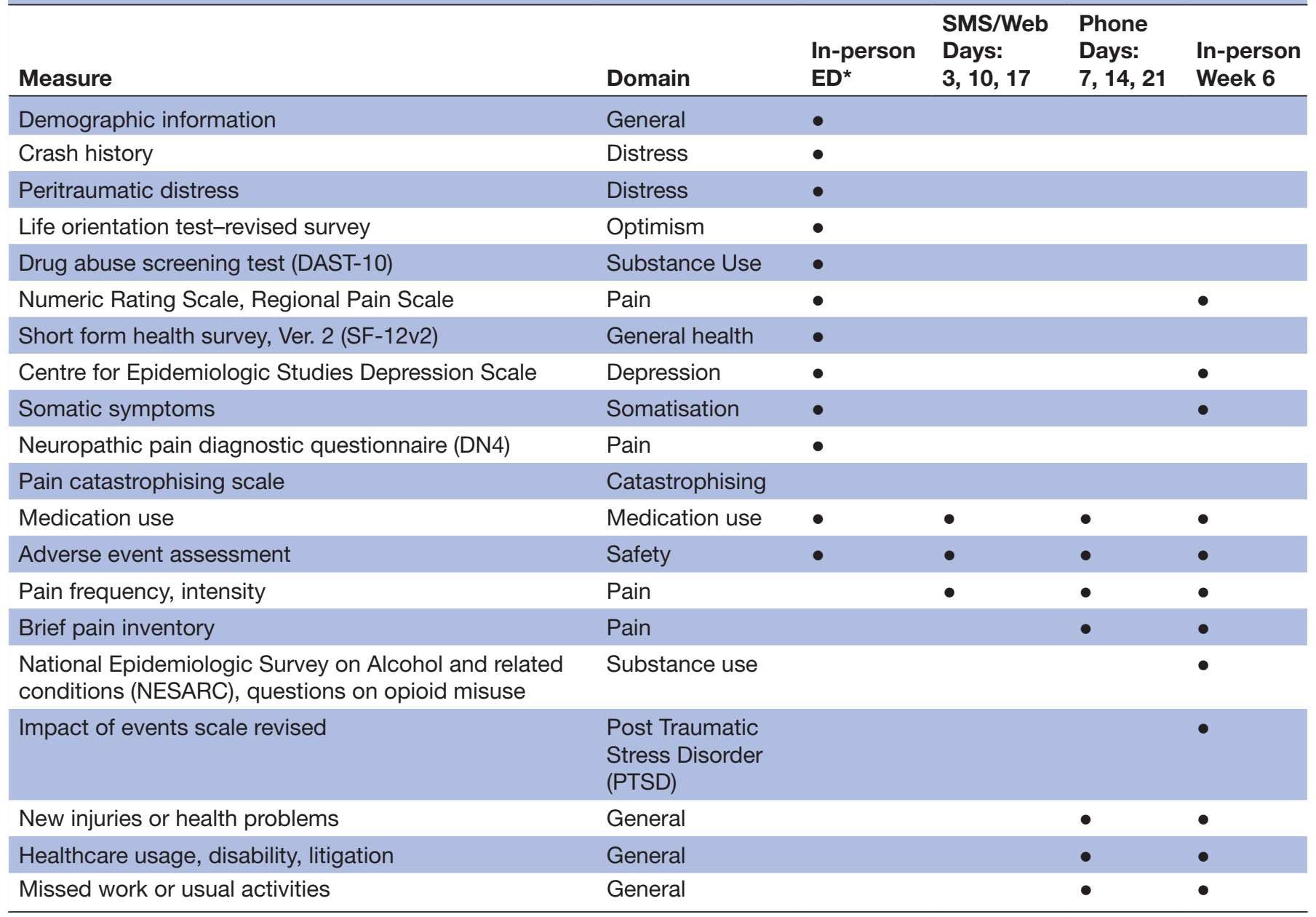

patients with mild severity (more likely to respond to placebo); minimising extraneous contact with investigative staff (perceived as therapeutic); and emphasising the concept of a placebo.

Following discharge, the patient will receive follow-up assessments via internet based surveys and phone to monitor for AEs and evaluate patient outcomes. The patient will also return to the study site for an in-person follow-up interview 6 weeks after their initial ED visit. Both the participant and interviewer will be blinded to randomisation. Participants reporting AEs may have their dosage advanced more slowly, or the next dose reduced or held as appropriate. If necessary, patients will be maintained on a lower dose, or their dose will be readvanced depending on the nature of the side effect(s). Patients who decline further participation and patients experiencing adverse effects that in the opinion of study investigators indicate that study drug should be stopped will be discontinued or tapered from study medication. Table 2 outlines the intended schedule of surveys and follow-up procedures after consent is obtained:

\section{Outcomes measured and statistical analyses}

Primary outcome

Overall tolerability will be assessed by (1) the proportion of participants in each treatment arm reporting adverse side effects and (2) the proportion of participants that drop-out due to side effects in the first 21 days of study assessments. Duloxetine will be considered acceptable if the drop-out rate differs by $\leq 25 \%$ in duloxetine arms versus the control arm and if the rate of AEs in the duloxetine treatment arms is $\leq 25 \%$ metrics chosen based on differences observed in numerous clinical trials of opioids and duloxetine conducted in patients with musculoskeletal pain. ${ }^{31}$ We will also explore adherence as a marker of tolerability. Adherence will be defined as successfully taking $\geq 70 \%$ of the study drug (average at least 5 out of 7 weekly doses) for the duration of the 2-week study drug protocol. Adherence will be determined by performing a pill count at the in-person visit at week 6. (If participants do have remaining medications, we will assist with safe medication disposal at the time of this visit.)

\section{Secondary outcomes}

1) Acute pain relief assessment: the measured outcome variable is self-reported axial pain (0-10 numeric rating scale (NRS)). These are obtained over time, and thus we will use mixed effects models with random intercepts to account for subject effects and the correlation among repeated measurements within a subject. The study is powered to detect a $33 \%$ difference in pain 
scores between groups, a change that has been previously established to represent a clinically important measurement in pain outcomes. ${ }^{32}$ Models will be developed which include as independent variables the treatment group and the time (day of the assessment). We will assess the effect of the intervention on acute pain (Days 3, 7, 10, 14, 17, 21, 42). The effect of the intervention on acute pain will be assessed by comparing the pain score slopes (pain decline) between the two groups in the model:

$$
P i j=\beta_{0}+\alpha_{0 \mathrm{i}}+\beta_{1} \text { Group }_{\mathrm{i}}+\beta_{2} \text { Day }_{\mathrm{i}}+\beta_{3}\left(\text { Group }_{\mathrm{i}} \times \text { Day }_{\mathrm{i}}\right)+\epsilon_{i j}
$$

Where $P \mathrm{ij}$ is the pain score for the ith individual at time point $\mathrm{j}, \beta \mathrm{s}$ denote fixed effects, $\alpha_{0 \mathrm{i}}$ is the random intercept for the ith individual and $\varepsilon$ corresponds to random errors. (both $\alpha$ and $\varepsilon$ are zero centred and normally distributed).

2) Persistent to moderate severe axial musculoskeletal pain: the measured outcome variable will be assessed at 6 weeks. Six weeks is a time of importance because chronic pain trajectories are established about 6 weeks after acute injury or reporting acute musculoskeletal pain (such as an MVC), ${ }^{33} 34$ allowing for greater statistical power in this pilot study-more participants are likely to complete follow-up at 6 weeks than 6 months. Moderate to severe axial musculoskeletal pain was defined as a pain score of $\geq 4$ out of 10 on the NRS in the back, neck or shoulders (axial) ${ }^{35}$ Moderate to severe axial musculoskeletal pain was chosen as the primary outcome because it is associated with risk for chronic pain development ${ }^{36}$ and because it of its clinical relevance. The presence of moderate to severe musculoskeletal pain correlates with other patient-centred outcome measures such impaired function. ${ }^{37}$ We will compare the proportion of participants with moderate to severe $(\mathrm{NRS} \geq 4)$ axial pain at week 6 ( $\chi^{2}$ test) between treatment groups. We will use a mixed model with random effects (as outlined above) to compare and determine the effect of treatment on musculoskeletal pain scores across time, up to week 6 , accounting for baseline pain scores and change in time.

3) Rescue analgesia use: we will use participant report of prescription opioids, NSAIDs and other treatments for pain. We will determine if participants have used opioids within the past week and past 24 hours. We will then further assess the type, dose, frequency and quantity taken on average in the past week and past 24 hours. For opioids, we will estimate average morphine equivalent daily dose.

\section{Sample size calculation}

For the primary aim, tolerability, it was determined that 12 participants per group would be required to detect a $25 \%$ difference in AEs (primary outcome), assuming a background rate of $10 \%$ AEs in the control group $(\alpha=0.05$ and $\beta=0.90$, two-sided test of proportions). To assess a $25 \%$ difference in the proportion of participants dropping out of the study, 20 participants per group is required, ( $\alpha=0.05$ and $\beta=0.90$, two-sided test of proportions). This assumes a drop-out rate in the control group of $33 \%$, typical of randomised controlled trials (RCTs). Therefore, a total of 60 participants will be recruited into the study ( $\mathrm{n}=20$ per arm). The sample size is not based on pain outcomes, as those are secondary endpoints in this pilot feasibility study.

\section{Monitoring}

The research committee of the University Emergency Medicine foundation will serve as the Data Safety Monitoring Board (DSMB). The DSMB will be used to review and approve the study protocol prior to the start of the study. It will conduct an unblinded analysis after 30 patients have conducted outcome assessments through day 21 and determine whether it is safe to continue the trial after this stage. In case of any serious adverse events (SAEs), the DSMB will convene.

The study principal investigator (PI) will monitor for AEs that change study risk level. If an $\mathrm{AE}$ occurs which changes the study risk level, the study PI will immediately report this event to the institutional review board, inform any coinvestigators, and oversee the process of modifying the study at the study site(s) as appropriate to address the change in risk. SAEs will be monitored by the study site investigative teams in real time throughout the trial.

\section{Patient and public involvement}

The patients and public were not involved in the planning of this study.

\section{Ethics and dissemination}

These results will be published in a peer reviewed scientific journal and presented at one or more scientific conferences.

\section{Author affiliations}

${ }^{1}$ Emergency Medicine, Rhode Island Hospital, Providence, Rhode Island, USA ${ }^{2}$ Biology and Medicine, Brown University, Providence, Rhode Island, USA ${ }^{3}$ Emergency Medicine and Anesthesiology, University of North Carolina, Chapel Hill, North Carolina, USA

${ }^{4}$ Emergency Medicine, Brown University Warren Alpert Medical School, Providence, Rhode Island, USA

${ }^{5}$ Health Services, Policy, and Practice, Brown University School of Public Health, Providence, Rhode Island, USA

Acknowledgements The authors would like to thank the study participants and the research assistants facilitating this study at the Rhode Island and Miriam Hospital Emergency Departments.

Contributors DRS and DHS contributed equally to this paper. DRS wrote the first draft of this protocol manuscript. DHS revised the first draft and listed the relevant final citations. FLB and SAM developed the protocol and received grant funding for the protocol. They revised and edited this manuscript. FLB takes responsibility for this work as a whole.

Funding The project described was supported by the MayDay Fund and an Institutional Development Award (U54GM115677) from the National Institute of General Medical Sciences of the National Institutes of Health, which funds Advance Clinical and Translational Research (Advance-CTR). The content is solely the responsibility of the authors and does not necessarily represent the official views of the National Institutes of Health.

Competing interests None declared.

Patient consent for publication Not required. 
Ethics approval This work was approved by the Institutional Review Board of the Miriam Hospital (Federal Wide Assurance FWA00003538).

Provenance and peer review Not commissioned; externally peer reviewed.

Open access This is an open access article distributed in accordance with the Creative Commons Attribution Non Commercial (CC BY-NC 4.0) license, which permits others to distribute, remix, adapt, build upon this work non-commercially, and license their derivative works on different terms, provided the original work is properly cited, appropriate credit is given, any changes made indicated, and the use is non-commercial. See: http://creativecommons.org/licenses/by-nc/4.0/.

\section{REFERENCES}

1. Verhaak PF, Kerssens JJ, Dekker J, et al. Prevalence of chronic benign pain disorder among adults: a review of the literature. Pain 1998;77:231-9.

2. Gaskin DJ, Richard P. The economic costs of pain in the United States. J Pain 2012;13:715-24.

3. Young Casey C, Greenberg MA, Nicassio PM, et al. Transition from acute to chronic pain and disability: a model including cognitive, affective, and trauma factors. Pain 2008;134:69-79.

4. McLean SA, Ulirsch JC, Slade GD, et al. Incidence and predictors of neck and widespread pain after motor vehicle collision among US litigants and nonlitigants. Pain 2014;155:309-21.

5. Sterling M. Identifying those at risk of developing persistent pain following a motor vehicle collision. J Rheumatol 2006;33:838-9.

6. Beaudoin FL, Gutman R, Merchant RC, et al. Persistent pain after motor vehicle collision: comparative effectiveness of opioids versus non-steroidal anti-inflammatory drugs prescribed from the emergency department-a propensity matched analysis. Pain 2016.

7. Friedman BW, Cisewski D, Irizarry E, et al. A randomized, doubleblind, placebo-controlled trial of naproxen with or without orphenadrine or methocarbamol for acute low back pain. Ann Emerg Med 2018;71:348-56.

8. Friedman BW, Irizarry E, Solorzano C, et al. Diazepam Is No Better Than Placebo When Added to Naproxen for Acute Low Back Pain. Ann Emerg Med 2017;70:169-76.

9. Hoppe JA, Kim H, Heard K. Association of emergency department opioid initiation with recurrent opioid use. Ann Emerg Med 2015;65:493-9.

10. Johnson SP, Chung KC, Zhong L, et al. Risk of Prolonged Opioid Use Among Opioid-Naïve Patients Following Common Hand Surgery Procedures. J Hand Surg Am 2016.

11. Linnstaedt SD, Hu J, Bortsov AV, et al. $\mu$-Opioid receptor gene a118 $\mathrm{g}$ variants and persistent pain symptoms among men and women experiencing motor vehicle collision. J Pain 2015;16:637-44.

12. Qadri YJ, Bortsov AV, Orrey DC, et al. Genetic polymorphisms in the dopamine receptor 2 predict acute pain severity after motor vehicle collision. Clin J Pain 2015;31:1.

13. Myers J, Wielage RC, Han B, et al. The efficacy of duloxetine, nonsteroidal anti-inflammatory drugs, and opioids in osteoarthritis: a systematic literature review and meta-analysis. BMC Musculoskelet Disord 2014;15:76.

14. Lunn MPT, Hughes RAC, Wiffen PJ. Cochrane Neuromuscular Group. Duloxetine for treating painful neuropathy, chronic pain or fibromyalgia. Cochrane Database Syst Rev 2014;50.

15. Sultan A, Gaskell H, Derry S, et al. Duloxetine for painful diabetic neuropathy and fibromyalgia pain: systematic review of randomised trials. BMC Neurol 2008;8:29.

16. Andrews JS, Wu N, Chen SY, et al. Real-world treatment patterns and opioid use in chronic low back pain patients initiating duloxetine versus standard of care. J Pain Res 2013;6:825-35.

17. Walderhaug E, Kasserman S, Aikins D, et al. Effects of duloxetine in treatment-refractory men with posttraumatic stress disorder. Pharmacopsychiatry 2010;43:45-9.
18. Jakupcak M, Luterek J, Hunt S, et al. posttraumatic stress and its relationship to physical health functioning in a sample of Iraq and Afghanistan War veterans seeking postdeployment VA health care. $J$ Nerv Ment Dis 2008;196:425-8.

19. Dobscha SK, Morasco BJ, Duckart JP, et al. Correlates of prescription opioid initiation and long-term opioid use in veterans with persistent pain. Clin J Pain 2013;29:102-8.

20. Jenewein J, Wittmann L, Moergeli H, et al. Mutual influence of posttraumatic stress disorder symptoms and chronic pain among injured accident survivors: a longitudinal study. J Trauma Stress 2009;22:n/a-8.

21. Hall JA, Wang F, Oakes TM, et al. Safety and tolerability of duloxetine in the acute management of diabetic peripheral neuropathic pain: analysis of pooled data from three placebo-controlled clinical trials. Expert Opin Drug Saf 2010;9:525-37.

22. Feinberg RK, Hu J, Weaver MA, et al. Stress-related psychological symptoms contribute to axial pain persistence after motor vehicle collision: path analysis results from a prospective longitudinal study. Pain 2017;158:682-90.

23. McGreevy K, Bottros MM, Raja SN. Preventing Chronic Pain following Acute Pain: Risk Factors, Preventive Strategies, and their Efficacy. Eur J Pain Suppl 2011;5:365-76.

24. King NS, Crawford S, Wenden FJ, et al. The Rivermead Post Concussion Symptoms Questionnaire: a measure of symptoms commonly experienced after head injury and its reliability. J Neurol 1995;242:587-92.

25. Radloff LS. The CES-D Scale: A self-report depression scale for research in the general population. Applied Psychological Measurement 1977;1:385-401.

26. Brunet A, Weiss DS, Metzler TJ, et al. The Peritraumatic Distress Inventory: a proposed measure of PTSD criterion A2. Am J Psychiatry 2001;158:1480-5.

27. Ware JE, Sherbourne CD. The MOS 36-item short-form health survey (SF-36). I. Conceptual framework and item selection. Med Care 1992;30:473-83.

28. Yudko E, Lozhkina O, Fouts A. A comprehensive review of the psychometric properties of the Drug Abuse Screening Test. J Subst Abuse Treat 2007;32:189-98.

29. Dworkin RH, Katz J, Gitlin MJ. Placebo response in clinical trials of depression and its implications for research on chronic neuropathic pain. Neurology 2005;65:S7-S19.

30. Price DD, Milling LS, Kirsch I, et al. An analysis of factors that contribute to the magnitude of placebo analgesia in an experimental paradigm. Pain 1999;83:147-56.

31. Abdel Shaheed C, Maher CG, Williams KA, et al. Efficacy, Tolerability, and Dose-Dependent Effects of Opioid Analgesics for Low Back Pain: A Systematic Review and Meta-analysis. JAMA Intern Med 2016;176:958-68.

32. Farrar JT, Berlin JA, Strom BL. Clinically important changes in acute pain outcome measures: a validation study. J Pain Symptom Manage 2003;25:406-11.

33. Sterling M, Hendrikz J, Kenardy J. Similar factors predict disability and posttraumatic stress disorder trajectories after whiplash injury. Pain 2011;152:1272-8.

34. Hu J, Bortsov AV, Ballina LE, et al. Chronic widespread pain after motor vehicle collision typically occurs via immediate development and non-recovery: Results of an emergency department-based cohort study. Pain 2015. In press.

35. Krebs EE, Carey TS, Weinberger M. Accuracy of the pain numeric rating scale as a screening test in primary care. J Gen Intern Med 2007;22:1453-8.

36. McLean SA, Clauw DJ, Abelson JL, et al. The development of persistent pain and psychological morbidity after motor vehicle collision: integrating the potential role of stress response systems into a biopsychosocial model. Psychosom Med 2005;67:783-90.

37. Sadosky AB, Taylor-Stokes G, Lobosco S, et al. Relationship between self-reported low-back pain severity and other patientreported outcomes: results from an observational study. J Spinal Disord Tech 2013;26:8-14. 